\title{
Low Temperature Electrical Transport in Double Layered CMR Manganite $\mathrm{La}_{1.2} \mathrm{Sr}_{1.4} \mathrm{Ba}_{0.4} \mathrm{Mn}_{2} \mathrm{O}_{7}$
}

\author{
Y. S. Reddy ${ }^{1}$, P. Kistaiah ${ }^{2}$, C. Vishnuvardhan Reddy ${ }^{2 *}$ \\ ${ }^{1}$ Department of Physics, Chaitanya Bharathi Institute of Technology, Gandipet, Hyderabad, India \\ ${ }^{2}$ Department of Physics, Osmania University, Hyderabad, India \\ Email: ${ }^{*}$ reddycvv@osmania.ac.in
}

Received 2012

\begin{abstract}
The electrical transport behavior and magnetoresistance (MR) of a polycrystalline double layered manganite $\mathrm{La}_{1.2} \mathrm{Sr}_{1.4} \mathrm{Ba}_{0.4} \mathrm{Mn}_{2} \mathrm{O}_{7}$, synthesized by the sol-gel method, are investigated in the temperature range $4.2 \mathrm{~K}-300 \mathrm{~K}$. The sample exhibits an insulator-to-metal transition at $87 \mathrm{~K}\left(\mathrm{~T}_{\mathrm{IM}}\right)$ and the spin-glass (SG)-like behavior is observed below $50 \mathrm{~K}\left(\mathrm{~T}_{\mathrm{SG}}\right)$. The transport behavior is analyzed in the entire temperature range considering three different regions: paramagnetic insulating region $\left(\mathrm{T}>\mathrm{T}_{\mathrm{IM}}\right)$, ferromagnetic metallic region $\left(\mathrm{T}_{\mathrm{SG}}<\mathrm{T}<\mathrm{T}_{\mathrm{IM}}\right)$ and antiferromagnetic insulating region $\left(\mathrm{T}<\mathrm{T}_{\mathrm{SG}}\right)$ by fitting the temperature dependent resistivity data to the equations governing the conduction process in the respective temperature regions. The results show that the conduction at $\mathrm{T}>\mathrm{T}_{\mathrm{IM}}$ follows Mott variable range hopping $(\mathrm{VRH})$ process, while the two-magnon scattering process is evidenced at $\mathrm{T}_{\mathrm{SG}}<\mathrm{T}<\mathrm{T}_{\mathrm{IM}}$ which is suppressed with the applied magnetic field of $4 \mathrm{~T}$. The low temperature conductivity data are also fitted with Mott VRH equation. The sample exhibits a large MR $\approx 45 \%$ ) over a temperature range $5 \mathrm{~K}-50 \mathrm{~K}$ and it shows $\approx 32 \% \mathrm{MR}$ at $5 \mathrm{~K}$ with a magnetic field of $0.5 \mathrm{~T}$.
\end{abstract}

Keywords: Layered Manganite; Magnetoresistance; Transport Behavior; Variable Range Hopping; Magnon Scattering

\section{Introduction}

The discovery of colossal magnetoresistance (CMR) in Labased double layered (DL) manganites $\mathrm{La}_{2-2 x} \mathrm{Sr}_{1+2 x} \mathrm{Mn}_{2} \mathrm{O}_{7}$ has provided an opportunity to explore the interaction among spin, charge and lattice in reduced dimensions [1,2]. These materials show large values of MR at moderate magnetic fields and proved to be promising materials for many technological applications. The $(\mathrm{La}, \mathrm{A})_{3} \mathrm{Mn}_{2} \mathrm{O}_{7}(\mathrm{~A}=\mathrm{Sr}, \mathrm{Ca}, \mathrm{Ba})$ perovskite compound with layered structure consists of the $\mathrm{MnO}_{2}$ bilayers which are respectively separated by the rock-salt-type (La,A) $\mathrm{O}_{2}$ layers along $c$-axis [3]. Because of its structural anisotropy, it is expected to present the anisotropy of physical, electrical and magnetic properties. Further, the natural array of conducting ferromagnetic/non-magnetic insulating/conducting ferromagnetic junctions present in the structure of these materials may lead to large CMR at low magnetic field, i.e., low field magnetoresistance [4]. Because of the reduced dimensionality, the balance between ferromagnetic double exchange ((FM-DE) and antiferromagnetic superexchange (AFM-SE) interactions between $\mathrm{Mn}$ ions is more subtle [5,6]. Therefore, one can expect that the slight changes in the size and/or concentration of (La,A) site ions can show significant effect on bulk transport and magnetic properties. Further, the Mn-O-Mn bond angle is about $180^{\circ}$ in the (La,A) ${ }_{3} \mathrm{Mn}_{2} \mathrm{O}_{7}$ system and is about $155-170^{\circ}$ in ( $\mathrm{La}, \mathrm{A}) \mathrm{MnO}_{3}$ system. The bond-length can be altered by the internal pressure, i.e., by changing the size and/or concentration of (La,A) site ions, however, the variation of the Mn-O-Mn bond-length in $\mathrm{Mn}_{2} \mathrm{O}_{7}$ system is different from that in $\mathrm{MnO}_{3}$ system $[7,8]$. Therefore, the study of lattice effects on the mag-

\footnotetext{
${ }^{*}$ Corresponding author.
}

netotransport properties in the $(\mathrm{La}, \mathrm{A})_{3} \mathrm{Mn}_{2} \mathrm{O}_{7}$ system might be useful in understanding the fundamentals of the CMR and its related properties.

We have prepared some DL manganite samples with different doping elements $\left(\mathrm{Ca}^{2+}, \mathrm{Ba}^{2+}\right)$ at $\mathrm{Sr}^{2+}$ site with different doping levels with an aim to increase $M R$ and $T_{I M}$ (insulator-to-metal transition temperature) and also to investigate the transport phenomena in these materials. In this paper, we present the results obtained for $\mathrm{La}_{1.2} \mathrm{Sr}_{1.4} \mathrm{Ba}_{0.4} \mathrm{Mn}_{2} \mathrm{O}_{7}$ which exists in three different regions: paramagnetic insulating region, ferromagnetic metallic region and antiferromagnetic insulating region in the temperature range $4.2 \mathrm{~K}-300 \mathrm{~K}$ with a main focus on its transport behavior.

\section{Experiment}

High pure powders of $\mathrm{La}_{2} \mathrm{O}_{3}, \mathrm{MnCO}_{3}, \mathrm{Sr}\left(\mathrm{NO}_{3}\right)_{2}$ and $\mathrm{Ba}\left(\mathrm{NO}_{3}\right)_{2}$. $4 \mathrm{H}_{2} \mathrm{O}$, in stoichiometric proportions, were used to obtain the nominal composition of $\mathrm{La}_{1.2} \mathrm{Sr}_{1.4} \mathrm{Ba}_{0.4} \mathrm{Mn}_{2} \mathrm{O}_{7} . \mathrm{La}_{2} \mathrm{O}_{3}$ and $\mathrm{MnCO}_{3}$ were converted into nitrates prior to use. All the nitrates were dissolved in the citric acid solution and then the $\mathrm{pH}$ was adjusted to 6 with ammonia solution. After getting the water evaporated from the solution, ethylene glycol was added to it and heated at about $90^{\circ} \mathrm{C}$ until a gel-type solution is formed. The gel was dried at $150^{\circ} \mathrm{C}$ and then decomposed at $250^{\circ} \mathrm{C}$ in air for $2 \mathrm{~h}$ to decompose nitrates and all organic materials. The resultant ash was ground to get a fine homogeneous powder. The powder was calcinated in air $1100^{\circ} \mathrm{C}$ for $10 \mathrm{~h}$ and then pressed into circular pellets. The pellets were finally sintered in air at $1400^{\circ} \mathrm{C}$ for $6 \mathrm{~h}$.

The structural characterization was carried out by X-ray diffraction using X-pert pro system, M/S Pananlytical $(\lambda=1.54056$ 
$\AA$ ) in the $\overparen{D}$ range $20^{\circ}-80^{\circ}$ with step size $0.01^{\circ}$ and a count time of $0.6 \mathrm{~s}$ per step. The results of powder X-ray diffraction suggest the formation of single phase with body-centered tetragonal structure (space group: I4/ $\mathrm{mmm}$ ). The electrical resistivity at different applied magnetic fields $(\mathrm{H}=0 \mathrm{~T}, 1.5 \mathrm{~T}$ and 4 $\mathrm{T}$ ) is measured by standard four-probe method over the temperature range $4.2 \mathrm{~K}-300 \mathrm{~K}$ with the use of a superconducting magnet system of Oxford.

\section{Results and Discussion}

The temperature (T) dependent electrical resistivity $(\rho)$ of the sample at different magnetic fields is shown in Figure 1. As the temperature is decreased from $300 \mathrm{~K}$, the resistivity of the sample increases and reaches maximum at $87 \mathrm{~K}$ which is known as insulator-to-metal transition temperature $\left(\mathrm{T}_{\mathrm{IM}}\right)$. As the temperature is further lowered from $\mathrm{T}_{\mathrm{IM}}$, an upturn of resistivity is observed at $\approx 50 \mathrm{~K}$ which is termed as spin-glass (SG)-like transition temperature $\left(\mathrm{T}_{\mathrm{SG}}\right)[7,8]$. The SG-like behavior is attributed to the competing intra-bilayer FM-DE and inter-bilayer AFM-SE interactions which usually coexist in quasi 2D bilayer manganites and become dominant at low temperatures. When magnetic field is applied, $\mathrm{T}_{\mathrm{IM}}$ shifts to higher temperatures whereas SG-like transition region gets broadened due to the suppression of magnetic fluctuations with the applied magnetic field.

The sample exists in three different states at different temperatures and hence to explain the nature of conduction mechanism of the sample, the temperature dependent electrical resistivity data are analyzed in the in the entire temperature range $(4.2 \mathrm{~K}-300 \mathrm{~K})$ in three different temperature regions: (i) paramagnetic (PM) insulating region $\left(T>T_{I M}\right)$, (ii) ferromagnetic (FM) metallic region $\left(\mathrm{T}_{\mathrm{SG}}<\mathrm{T}<\mathrm{T}_{\mathrm{IM}}\right)$ and (iii) antiferromagentic (AFM) insulating region $\left(\mathrm{T}<\mathrm{T}_{\mathrm{SG}}\right)$.

\subsection{Conduction Mechanism at $T>T_{I M}$}

The conduction mechanism in PM semiconducting/insulating region in manganites is usually explained by four models: They are: (i) semiconduction (SC) model described by Arrhenius equation $\rho=\rho_{0} \exp \left(E_{a} / k_{B} T\right)$ [9], (ii) nearest neighbor small polaron hopping $(\mathrm{SPH})$ model described by $\rho=\rho_{0} \mathrm{~T}^{\mathrm{n}} \exp \left(\mathrm{E}_{\mathrm{p}} / \mathrm{k}_{\mathrm{B}} \mathrm{T}\right)$,

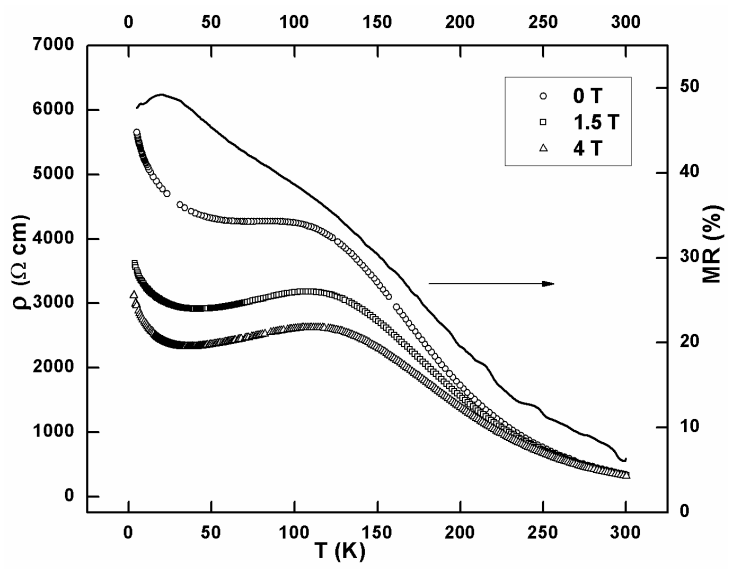

Figure 1. Temperature dependent Electrical resistivity and MR plots of $\mathrm{La}_{1.2} \mathrm{Sr}_{1.4} \mathrm{Ba}_{0.4} \mathrm{Mn}_{2} \mathrm{O}_{7}$. where $\mathrm{n}=1$ for adiabatic hopping [10] and $\mathrm{n}=1.5$ for nonadiabatic hopping [11], (iii) Mott type of VRH model described by $\rho=\rho_{\infty} T^{n} \exp \left(T_{0} / T\right)^{p}$, where $p=1 /(d+1)$, $d$ being the dimensionality of the system [12,13] and (iv) Efros-Shkloskii (ES) type of VRH model described by [14]. The value of characteristic temperature $\left(T_{0}\right)$ in Mott VRH model is given by $24 / \pi$ $\mathrm{L}^{\mathrm{d}} \mathrm{k}_{\mathrm{B}} \mathrm{N}\left(\mathrm{E}_{\mathrm{F}}\right)$, where $\mathrm{L}$ is localization length of trapped charge carriers (here, $\left.L=10^{-10} \mathrm{~m}\right), \mathrm{N}\left(\mathrm{E}_{\mathrm{F}}\right)$ is density of the localized states at Fermi level and $d$ is the dimensionality of the system. The Coulomb interaction in hopping regime which produces a gap in electronic density of states (DOS) is responsible for ES VRH type of conduction mechanism, whereas Mott VRH arises when such gap is filled. Each model predicts a different temperature dependence of the resistivity and fits the resistivity data in different temperature ranges.

In the present study, the $\rho$-T data are analyzed by fitting the data to all the equations mentioned above. The $\rho$-T data do not fit well to the equations of SC and SPH models; ES VRH gives reasonably good fittings, but the best fittings are obtained with Mott VRH model over a wide temperature range (Figure 2). The Mott 2D and 3D VRH models give almost indistinguishable fittings for drawing any conclusion about dimensionality dependence, however, the results clearly point towards Mott type of VRH conduction mechanism in PM insulating region $\left(\mathrm{T}>\mathrm{T}_{\mathrm{IM}}\right)$. The best fit parameters obtained with Mott 2D and 3D VRH models are listed in Table $\mathbf{1}$ and they are in good agreement with the previous reports on similar DL manganites $[15,16]$. The decrease in the values of $T_{0}$ and the increase in the values of $\mathrm{N}\left(\mathrm{E}_{\mathrm{F}}\right)$ with the magnetic field is due to the suppression of magnetic domain scattering with applied magnetic field.

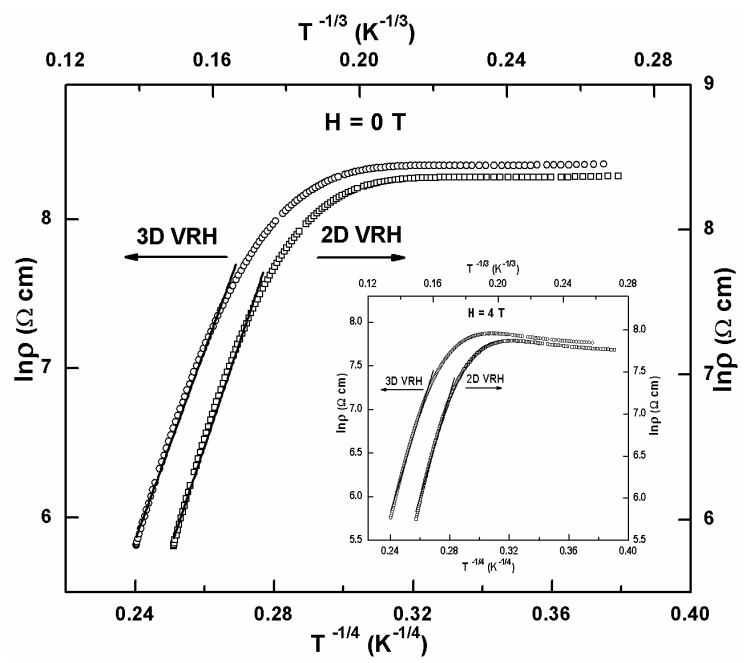

Figure 2. Plots of $\ln \rho-T^{-1 / 4}$ and $\ln \rho-T^{-1 / 3}$ for $\operatorname{La}_{1.2} \mathrm{Sr}_{1.4} \mathrm{Ba}_{0.4} \mathrm{Mn}_{2} \mathrm{O}_{7}$. The solid lines give the best fits to Mott $2 \mathrm{D}$ and $3 \mathrm{D}$ VRH models.

Table 1. The best fit parametres obtained from mott 2d and 3d vrh model fittings.

\begin{tabular}{|c|c|c|c|c|c|c|}
\hline \multirow{2}{*}{$\begin{array}{c}\mathbf{H} \\
(\mathrm{T})\end{array}$} & \multicolumn{3}{|c|}{ Mott 3D VRH } & \multicolumn{3}{|c|}{ Mott 2D VRH } \\
\hline & $\begin{array}{c}\rho_{0} \\
(\Omega \mathrm{cm})\end{array}$ & $\begin{array}{c}T_{0} \\
(K)\end{array}$ & $\begin{array}{c}N\left(E_{F}\right) \\
\left(e V^{1} c^{-3}\right)\end{array}$ & $\begin{array}{c}\rho_{0} \\
(\Omega \mathrm{cm})\end{array}$ & $\begin{array}{c}T_{0} \\
(K)\end{array}$ & $\begin{array}{c}N\left(E_{F}\right) \\
\left(e V^{-1} \mathrm{~cm}^{-2}\right)\end{array}$ \\
\hline 0 & $8.3 \times 10^{-5}$ & $1.6 \times 10^{7}$ & $5.4 \times 10^{21}$ & $4.7 \times 10^{-3}$ & $4.3 \times 10^{5}$ & $2.1 \times 10^{15}$ \\
\hline 4 & $4.5 \times 10^{-4}$ & $1.0 \times 10^{7}$ & $8.8 \times 10^{21}$ & $1.5 \times 10^{-2}$ & $3.0 \times 10^{5}$ & $2.9 \times 10^{15}$ \\
\hline
\end{tabular}




\subsection{Conduction Mechanism at $T_{S G}<T<T_{I M}$}

The electron transport mechanism in the FM metallic region is usually understood by fitting the resistivity data to a general Zener-Double Exchange polynomial law $\rho=\rho_{0}+\rho_{2} T^{2}+\rho_{n} T^{n}$, where $\rho_{0}$ is the residual resistivity and is independent of temperature, $\rho_{2}$ is the resistivity contributed by electronelectron and electron-phonon scattering mechanisms and $\rho_{n}$ is the resistivity coefficient corresponding to $n$, which takes values from 2.5 to 7.5 [14,17]. The value of $n$ is included by taking spin fluctuations into account. Further, the low value of $\mathrm{n}(<4.5)$ corresponds to one-magnon scattering process, whereas the high value of $\stackrel{\mathbf{4}}{\mathbf{4} .5}$ ) corresponds to two-magnon scattering process.

The transport behavior at $\mathrm{T}<\mathrm{T}_{\mathrm{IM}}$ in polycrystalline bilayer manganites has not been studied much unlike the transport mechanism at $\mathrm{T}>\mathrm{T}_{\mathrm{IM}}$ in layered manganites. Zhang et al. [6] found $\mathrm{T}^{9 / 2}$ dependence in single crystals of $\mathrm{La}_{1.2} \mathrm{Sr}_{1.8} \mathrm{Mn}_{2} \mathrm{O}_{7}$, but they did not include $\rho_{2} \mathrm{~T}^{2}$ term. Therefore, an attempt is made to explore the nature of transport behavior at $\mathrm{T}_{\mathrm{SG}}<\mathrm{T}<\mathrm{T}_{\mathrm{IM}}$ in the present DL manganite sample.

In the absence of magnetic field, the FM metallic region is very small and hence we fitted the $\rho$-T data $(H=1.5 \mathrm{~T}, 4 \mathrm{~T})$ in the temperature region $45 \mathrm{~K}-95 \mathrm{~K}$ with Zener DE polynomial law (Figure 3). The $\rho$ - $\mathrm{T}$ data $(\mathrm{H}=1.5 \mathrm{~T})$ are well fitted with Zener DE polynomial law for $n=4.5$ indicating the two- magnon scattering contribution to the conductivity along with electron-electron and electron-phonon scattering mechanisms. The $\rho$ - $\mathrm{T}$ data $(\mathrm{H}=4 \mathrm{~T})$ are nicely fitted with $\rho=\rho_{0}+\rho_{2} \mathrm{~T}^{2}$ suggesting the suppression of spin fluctuations with the magnetic field and the conduction in this region is mainly due to electron-electron and electron-phonon interactions [14]. The obtained best-fit parameters are: $\rho_{0}=2826.11 \Omega \mathrm{cm}, \rho_{2}=0.0365$ $\Omega \mathrm{cm} \mathrm{K}^{-2}$ and $\rho_{4.5}=3.84 \times 10^{-9}(\mathrm{H}=1.5 \mathrm{~T})$ and $\rho_{0}=2272.80 \Omega$ $\mathrm{cm}, \rho_{2}=0.0369 \Omega \mathrm{cm} \mathrm{K}^{-2}(\mathrm{H}=4 \mathrm{~T})$. The applied magnetic field can decrease the magnetic domain boundary and therefore $\rho_{0}$ decreases.

\subsection{Conduction Mechanism at $T<T_{S G}$}

The low temperature upturn of resistivity is a typical characteristic of DL manganites. The transport behavior of bilayer manganites in $\mathrm{AFM}$ insulating region $\left(\mathrm{T}<\mathrm{T}_{\mathrm{SG}}\right)$ is very interesting

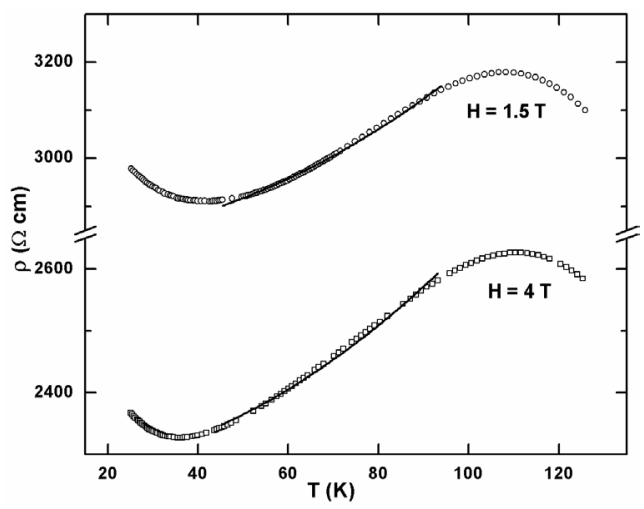

Figure 3. Temperature versus resistivity plots of $\mathbf{L a}_{1.2} \mathbf{S r}_{1.4} \mathbf{B a}_{0.4}$ $\mathbf{M n}_{2} \mathbf{O}_{7}$. The solid lines give the best fits to $\rho=\rho_{0}+\rho_{2} \mathbf{T}^{2}+\rho_{4.5} \mathbf{T}^{4.5}(\mathbf{H}$ $=1.5 \mathrm{~T})$ and $\rho=\rho_{0}+\rho_{2} \mathrm{~T}^{2}(\mathrm{H}=4 \mathrm{~T})$. and worthy of study. Zhu et al. [18] have found the band transport process, Zhang et al. [6] have showed that conductivity is proportional to $\mathrm{T}^{1 / 2}$ and Zhang et al. [19] fitted the upturn of resistivity using Mott VRH equation in similar DL manganites.

To explore the nature of conduction mechanism in AFM insulating phase, the $\sigma-\mathrm{T}$ data at $\mathrm{T}<\mathrm{T}_{\mathrm{SG}}$ are fitted to all the equations mentioned in section 3.A. and $\mathrm{T}^{1 / 2}$ dependence of conductivity is also examined. The $\mathrm{T}^{1 / 2}$ dependence of conductivity is a characteristic of weak localization effects in 3D disordered metals and indicate the contribution of electron-electron interactions to the conductivity. The best fittings are obtained with Mott VRH suggesting that the conduction at $\mathrm{T}<\mathrm{T}_{\mathrm{SG}}$ is also governed by Mott VRH law (Figure 4). Here also, Mott 2D VRH and Mott 3D VRH equations give almost indistinguishable fittings and hence it is difficult to draw any conclusion about dimensionality dependence.

\subsection{Magnetoresistance}

In Figure 1, the right panel shows the variation of $M R(H=4 T)$ with temperature. The MR - T curve displays no peak at $T_{I M}$ unlike the peak displayed at $\mathrm{T}_{\mathrm{IM}}$ by resistivity curves and this is a special feature of DL manganites [7,8]. The sample shows $\approx 40 \% \mathrm{MR}$ at its $\mathrm{T}_{\mathrm{IM}}$ and the maximum $\mathrm{MR}$ is $\approx 50 \%$ at $\approx 20 \mathrm{~K}$. It is noteworthy that the sample exhibits $\approx 45 \% \mathrm{MR}$ in the te $\mathrm{m}$ perature range $5 \mathrm{~K}-50 \mathrm{~K}$. This property of exhibiting CMR effect over a wide temperature region supplies the potential applications for layered perovskites.

The variation of MR with applied magnetic field at $5 \mathrm{~K}$ and $90 \mathrm{~K}$ is shown in Figure 5. The increase of MR with magnetic field is rapid at $5 \mathrm{~K}$ than that at $90 \mathrm{~K}$ which suggests that the suppression of magnetic frustration and spin scattering with applied magnetic field is more in SG-like region than that near the vicinity of $\mathrm{T}_{\mathrm{IM}}$. The striking feature from these curves is that the sample shows $\approx 32 \% \mathrm{MR}$ at $5 \mathrm{~K}$ with applied magnetic field of $0.5 \mathrm{~T}$ which is indeed a sign of low field magnetoresistance.

\section{Conclusions}

In conclusion, a DL manganite sample $\mathrm{La}_{1.2} \mathrm{Sr}_{1.4} \mathrm{Ba}_{0.4} \mathrm{Mn}_{2} \mathrm{O}_{7}$ is investigated with respect to its $\mathrm{MR}$ and electrical transport behavior in the temperature range $4.2 \mathrm{~K}-300 \mathrm{~K}$. The conduction

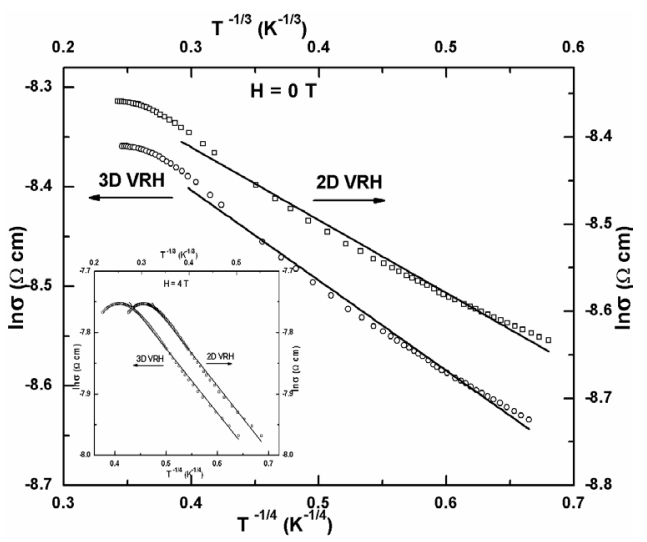

Figure 4. Plots of $\ln \rho-\mathrm{T}^{-1 / 4}$ and $\ln \rho-\mathrm{T}^{-1 / 3}$ for $\mathrm{La}_{1.2} \mathrm{Sr}_{1.4} \mathrm{Ba}_{0.4} \mathrm{Mn}_{2} \mathrm{O}_{7}$. The solid lines give the best fits to Mott $2 \mathrm{D}$ and $3 \mathrm{D}$ VRH models. 


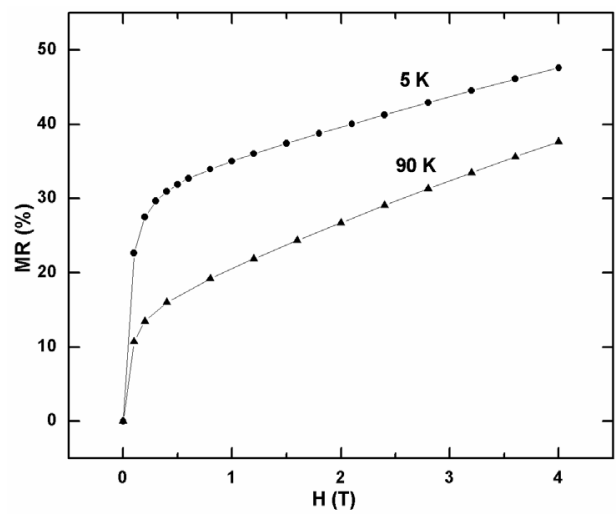

Figure 5. The variation of MR with appline magnetic field for $\mathbf{L a}_{1.2} \mathrm{Sr}_{1.4} \mathbf{B a}_{0.4} \mathrm{Mn}_{2} \mathrm{O}_{7}$.

process at $\mathrm{T}>\mathrm{T}_{\mathrm{IM}}$ is due to Mott VRH process and the metallic conduction is contributed by electron-electron scattering and two-magnon scattering at low magnetic fields and at higher fields magnon scattering mechanism is disappeared. The conductivity in SG-like region is also governed by Mott VRH process. The property of exhibiting large MR over a wide temperature range and low field magnetoresistance are found in this sample.

\section{Acknowledgements}

The authors are thankful to the centre director, UGC-DAE Consortium for Scientific Research, Indore, for providing experimental facilities.

\section{REFERENCES}

[1] Y. Moritomo, A. Asamitsu, H. Kuwahara, and Y. Tokura, "Giant magnetoresistance of manganese oxides with a layered perovskite structure”, Nature (London), vol. 380, pp. 141-144, March 1996.

[2] Y. Moritomo, A. Asamitsu, H. Kuwahara, and Y. Tokura, "Giant magnetoresistance of manganese oxides with a layered perovskite structure”, Nature (London), vol. 380, pp. 141-144, March 1996.

[3] T. Kimura, Y.Tomioka, H. Kuwahara, A. Asamitsu, M.Tamura, and Y.Tokura, "Interplane tunneling magnetoresistance in a layered manganite crystal”, Science, vol. 274, pp. 1698 1701, December 1996.

[4] Hong Zhu, XianMing Liu, KeQing Ruan, and YuHeng Zhang, "Magnetic inhomogeneity and variable-range hopping transport at temperatures above the ferromagnetic transition in $\mathrm{La}_{1.4} \mathrm{Sr}_{1.6} \mathrm{Mn}_{2-\mathrm{y}} \mathrm{Ti}_{\mathrm{y}} \mathrm{O}_{7}$ system”, Phys. Rev. B,vol. 65, pp. 104424 (1-7), February 2002.

[5] E. O. Chi, Y.-U. Kwon, J.-T. Kim, and N. H. Hur, "Lattice effects on the magnetic and transport properties in $\mathrm{La}_{1.4} \mathrm{Sr}_{1.6-x} \mathrm{~A}_{x} \mathrm{Mn}_{2} \mathrm{O}_{7}(\mathrm{~A}=\mathrm{Ca}, \mathrm{Ba})$ ), Solid State Commun., vol. 110, pp. 569, 1999.

[6] S. Okamoto, S. Ishihara, and S. Maekawa, "Orbital structure and magnetic ordering in layered manganites: Universal correlation and its mechanism”, Phys. Rev. B, vol. 63, pp. 104401 (1-6),
February 2001.

[7] C. L. Zhang, X. J. Chen, C. C. Almasan, and J. S. Gardner, J. L. Sarrao, "Low-temperature electrical transport in bilayer manganite $\mathrm{La}_{1.2} \mathrm{Sr}_{1.8} \mathrm{Mn}_{2} \mathrm{O}_{7}$ ”, Phys. Rev. B, vol. 65, pp. 134439 (1-6), March 2002.

[8] S. Chatterjee, P. H. Chou, C. F. Chang, I. P. Hong, and H. D. Yang, "Lattice effects on the transport properties of (R,Sr) ${ }_{3} \mathrm{Mn}_{2} \mathrm{O}_{7}(\mathrm{R}=\mathrm{La}, \mathrm{Eu}, \mathrm{Pr})$ ”, Phys. Rev. B, vol. 61, pp. 6106 -6113, March 2000.

[9] H. Zhu, D. Zhu, and Y. Zhang, "Effect of lattice expansion on the magnetotransport properties in layered manganites $\mathrm{La}_{1.4} \mathrm{Sr}_{1.6-\mathrm{y}} \mathrm{Ba}_{\mathrm{y}} \mathrm{Mn}_{2} \mathrm{O}_{7}$ ”, J. Appl. Phys., vol. 92, pp. 7355 - 7361, December 2002.

[10] S. B. Ogale, V. Talyansky, C. H. Chen, R. Ramesh, R. L. Green, and T. Venkatesan, "Unusual electric field effects in $\mathrm{Nd}_{0.7} \mathrm{Sr}_{0.3} \mathrm{MnO}_{3}$ ”, Phys. Rev. Lett., vol. 77, pp. 1159 - 1162, August 1996.

[11] G. Jeffrey Snyder, R. Hiskes, S. Dicarolis, M. R. Beasley, and T. H. Geballe, "Intrinsic electrical transport and magnetic properties of $\mathrm{La}_{0.67} \mathrm{Ca}_{0.33} \mathrm{MnO}_{3}$ and $\mathrm{La}_{0.67} \mathrm{Sr}_{0.33} \mathrm{MnO}_{3}$ MOCVD thin films and bulk material”, Phys. Rev. B, vol. 53, pp. 14434 - 14444, June 1996.

[12] M. Jaime, H.T. Hardner, M.B. Salamon, M. Rubinstein, P. Dorsey, and D. Emin, "Hall-effect sign anomaly and small-polaron conduction in $\left(\mathrm{La}_{1-\mathrm{x}} \mathrm{Gd}_{\mathrm{x}}\right)_{0.67} \mathrm{Ca}_{0.33} \mathrm{MnO}_{3}$ ”, Phy. Rev. Lett., vol. 78, pp. 951-954, February 1997.

[13] M. Viret, L. Ranno, and J. M. D. Coey, "Colossal magnetoresistance of the variable range hopping regime in the manganites", J Appl. Phys., vol. 81, pp. 4964 - 4966, April 1997.

[14] Yu Wang and Jorge J. Santiago-Aviles, "Large negative magnetoresistance and strong localization in highly disordered electrospun pregraphitic carbon nanofiber”, Appl. Phys. Lett., vol. 89, pp. 123119 (1-3), September 2006.

[15] D. S. Rana, C. M. Thaker, K. R. Mavani, D. G. Kuberkar, Darshan C. Kundaliya, and S. K. Malik, "Magnetic and transport properties of $\left(\mathrm{La}_{0.7-2 \mathrm{x}} \mathrm{Eu}_{\mathrm{x}}\right)\left(\mathrm{Ca}_{0.3} \mathrm{Sr}_{\mathrm{x}}\right) \mathrm{MnO}_{3}$ : Effect of simultaneous size disorder and carrier density”, J. Appl. Phys., vol. 95, pp. 4934 - 4940, May 2004.

[16] M. Matsukawa, M. Chiba, E. Kikuchi,R. Suryanarayanan, M. Apostu, S. Nimori, K. Sugimoto, and N. Kobayashi, "Effect of suppression of local distortion on the magnetic, electrical, and thermal transport properties of the Cr-substituted bilayer manganite $\mathrm{LaSr}_{2} \mathrm{Mn}_{2} \mathrm{O}_{7}$ ”, Phys. Rev. B, vol. 72, pp. 224422 (1-8), December 2005.

[17] M. H. Ehsani, P.Kameli, and M. E.Ghazi, "Influence of grain size on the electrical properties of the double-layered $\mathrm{LaSr}_{2} \mathrm{Mn}_{2} \mathrm{O}_{7}$ manganite”, J. Phys. Chem. Sol., vol. 73, pp. 744-750, 2012.

[18] Aritra Banerjee, S. Pal, and B. K. Chaudhuria, "Nature of small-polaron hopping conduction and the effect of $\mathrm{Cr}$ doping on the transport properties of rare-earth manganite $\mathrm{La}_{0.5} \mathrm{~Pb}_{0.5} \mathrm{Mn}_{1-\mathrm{x}} \mathrm{Cr}_{\mathrm{x}} \mathrm{O}_{3}$ ”, J. Chem. Phys., vol. 115, pp. 1550-1558, July 2001.

[19] Hong Zhu, XiaoJun $\mathrm{Xu}, \mathrm{Li} \mathrm{Pi}$, and YuHeng Zhang, " Two-dimensional magnetic correlation and transport behavior of layered manganite $\mathrm{La}_{1.4} \mathrm{Sr}_{1.6} \mathrm{Mn}_{2-\mathrm{x}} \mathrm{Cu}_{x} \mathrm{O}_{7}$ ”. Phys. Rev. B, vol. 62, pp. 6754-6760, September, 2000.

[20] R. L. Zhang, W. H. Song, Y. Q. Ma, J. Yang, B. C. Zhao, Z. G. Sheng, J. M. Dai, and Y. P. Sun, "Influence of Co doping on the charge-ordering state of the bilayered manganite $\mathrm{LaSr}_{2} \mathrm{Mn}_{2} \mathrm{O}_{7}$ ", Phys. Rev. B., vol. 70, pp. 224418 (1-6), December 2004. 